\title{
A Study on Tacit Knowledge Sharing in ERP Enterprises
}

\author{
Binli Sun \\ School of Economic Management, Beijing Institute of Petrochemical Technology, Beijing \\ 102617, P.R. China sunbinli@bipt.edu.cn
}

\begin{abstract}
With the fast developing knowledge economy, one of the major challenges facing enterprise managers is aligning knowledge management (KM) with business strategy and processes, especially after an enterprise has applied Enterprise Resource Planning (ERP). Since knowledge sharing $(\mathrm{KnS})$ is one of the most critical steps in KM activities, it is necessary to study the effective sharing of tacit knowledge in ERP enterprises. In an ERP environment, $\mathrm{KnS}$ system should have two functions. One is to support the enterprise business and the other is to support employees' communication. Most researches have thus far focused on the first function trying to find out management mechanism to improve the effect of $\mathrm{KnS}$ while ignoring the other function. In the $\mathrm{KnS}$ with the communication function, this research finds out that knowledge is exchanged naturally and freely. The $\mathrm{KnS}$ system can also make employees feel better about their work by making greater contribution to building an active culture environment to support knowledge exchange. In this paper, these two types of $\mathrm{KnS}$ are called business-oriented knowledge-sharing and subject-oriented knowledge-sharing. It also designs their constructions, propose their management mechanism and discuss some relevant practice problems. When these two types of $\mathrm{KnS}$ are applied in ERP enterprises they not only can provide channels for employees to exchange knowledge, but can also help enterprises to get tacit knowledge from ERP users and to make tacit knowledge more effectively be converted to explicit knowledge. Though ERP and $\mathrm{KnS}$ are performed as two separate systems, this research provides guidance to enterprise managers to develop tacit knowledge-sharing system on their ERP implementation and keep the same goal with it.
\end{abstract}

Keywords: Knowledge management, Enterprise resource planning (ERP), Enterprise systems

\section{INTRODUCTION}

In an ever-flattening world, organizations must quickly learn and adapt to changes in the marketplace. While we have become better at implementing new business processes and new management information system, such as ERP, we found that we spent too much time on cyber-business line and lost many chances to communicate with our partners. Nowadays, the end users are more involved in ERP implementations than they were in their previous traditional business process [1].

Please use the following format when citing this chapter:

Sun, B., 2007, in IFIP International Federation for Information Processing, Volume 254. Research and Practical Issues of Enterprise Information Systems II Volume 1, eds. L. Xu. Tjoa A.. Chaudhry S. (Boston: Springer), pp. 763-770. 
To make matter worse, ERP requires the end users to have more divergent knowledge. However, there is often a large gap in knowledge among ERP users, and they do not easily share what they know during their daily work [2]. Some researches have paid attention to the long-term impact of ERP implementation in organizations, and emphasized the importance of organizational learning in ERP implementation [3]. In ERP environment, enterprises will have greater challenges in knowledge management, especially those challenges related to the sharing and integration of knowledge. It is necessary, therefore, to discuss how to build up an effective knowledge sharing models in enterprises. When these models are put into practice, it will improve the organizational learning, knowledge exchange, tacit externalization, and to enhance organizational competitive edge.

In addition of the Introduction Section, the remainder of this paper is organized as follows: Section II provides an analysis to functions and types of knowledge sharing in ERP enterprises. Section III provides the construction of business-oriented $\mathrm{KnS}$ and its further analysis. Discussions about the subject-oriented $\mathrm{KnS}$ are presented in Section IV. Finally, Section V is the conclusions.

\section{FUNCTIONS AND TYPES OF KNOWLEDGE SHARING IN ERP ENTERPRISES}

ERP implementation relates closely to knowledge management in manufacturing enterprises. An ERP system stores a company's data, processes its information, and embeds its knowledge in business activities. Such knowledge may reside in company database such as explicit transaction knowledge. Some knowledge such as the process knowledge is embedded in the business activities. Other knowledge may be recorded in process manuals on a regular basis [4]. In addition, there is much more knowledge embedded in the heads of individuals who work directly with the ERP systems. The knowledge stored in database is explicit knowledge -- a kind of formal knowledge, and it can support the employees' work according to certain knowledge management process. Most of the knowledge stored in individuals' heads is tacit knowledge -- an informal knowledge, and it can only be used by communication among concerned people.

\subsection{Functions of Knowledge Sharing in ERP Enterprises}

The functions of knowledge management are often described as a knowledge support system to personnel in an enterprise. They provide intelligence to knowledge analysis and employees' interests and behavior in support of personalized access to the knowledge base [5]. This type of knowledge sharing is often described as value knowledge exchanging in a certain community, such as seeking and giving advice, sharing individual experience, new solutions to some problems, and supporting each other's work, and so on. To make these functions effective there is a need to research the motivation of $\mathrm{KnS}$, and evaluation mechanism and some external rewards [6]. 
In ERP enterprises, beside the functions of $\mathrm{KnS}$ discussed above, we should notice that $\mathrm{KnS}$ in ERP enterprise should have another function, which is to provide a way for ERP users to communicate with each others freely. In this way, employees can exchange their idea, their feeling, their questions and anything which they are interested in. Communicating to other people is a common human behavior. This kind of $\mathrm{KnSs}$ dose not need much more external rewards. It has been used in Lenovo company in China and has received a good evaluation from employees.

In general, we can see that there are two major functions of $\mathrm{KnS}$ in ERP enterprises, one is a working-support function, and the other is a daily communicating-support function. They are two sides of $\mathrm{KnS}$ in ERP enterprises.

\subsection{Types of Knowledge Sharing in ERP Enterprises}

In traditional enterprises, only small circles of colleagues and work groups commonly share their tacit knowledge, but in an ERP system, if we can get support from IT technology, the circles of colleagues and work groups sharing knowledge have been greatly extended. Such a sharing process is also more complex. According to the functions discussed above, we found the two functions of $\mathrm{KnS}$ need different support mechanism. The working-support $\mathrm{KnS}$ is commonly related to problems that employees met or discovered in their daily work. These problems have to be solved quickly so that the effect of $\mathrm{KnS}$ application will influence the ERP business and enterprise operations. To do this well some kinds of encourage mechanisms is needed to insure the system can work effectively. The communication-support $\mathrm{KnS}$ is commonly related to activities that employees' took part during their daily life. This type of activities has a wider scope which may include their work, their feeling and their life. This communication does not need some special encouraging mechanism. The practice methods are different between the two types of $\mathrm{KnS}$. In order to easily discuss $\mathrm{KnSs}$, we divided them into two types: one is business-oriented knowledgesharing, and the other is subject-oriented knowledge-sharing. We will discuss each of them in following sections.

\section{BUSINESS-ORIENTED KNOWLEDGE-SHARING MODEL}

The knowledge framework used in the business-oriented knowledge-sharing model is same as the framework in ERP. This means that the content of knowledge is similar to the ERP business. The managers and ERP line workers use the business function modules to finish their daily work. When this kind of $\mathrm{KnS}$ system is used on the internal platform, workers will quickly understand the knowledge framework, without much more training, because they have much more knowledge already about the ERP business. Such a KnS system can facilitate the process of knowledge sharing and reduce the time of training. When related materials and Q\&As in one business field can be collected together, it will be of great convenience for the employees to discuss the similar questions and promote the efficiency in solving problems. 


\subsection{Construction of Business-oriented Knowledge-sharing Model}

Business-oriented knowledge communication is similar to a kind of formal communication. There is a lot of knowledge exchange under this framework of $\mathrm{KnS}$. Workers in an enterprise can load up some materials, such as words, audio, and video materials, and store them in knowledge database after treatment. Workers can write anything that is related with their work and load-up to knowledge center. The tacit knowledge that cannot be expressed clearly can be recorded or kinescoped as audio or video materials and load-up in an enterprise's knowledge database. Question and answer method is another important channel for workers to exchange their knowledge. By asking and answering, worker can get the solution to the problem occurred in their work. In this way, $\mathrm{KnS}$ can get more knowledge from their workers. Meanwhile, Q\&A often focuses on the difficult area in employees' work, so the knowledge came from this channel is more important than the knowledge from subject-oriented $\mathrm{KnS}$. Generally, we need certain managers who know the enterprise very well to process the knowledge, such as select the valuable knowledge and give deep treatment before storing. The logic construction of business-oriented knowledgesharing model is shown in fig. 1 .

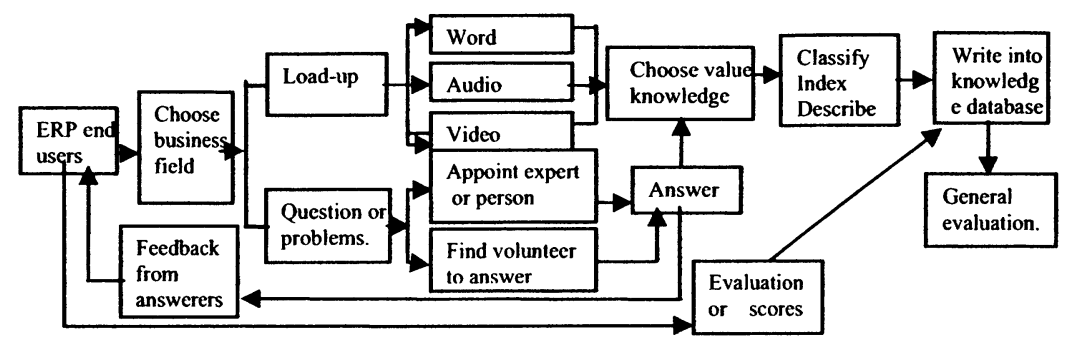

Figure 1. Business-oriented KnS Process Model

\subsection{Classification and Restricted Using}

In order to keep a close relationship between knowledge and ERP business, business-oriented $\mathrm{KnS}$ system should be divided into different fields that are fit to different ERP business modules. ERP end users choose related field to load up their knowledge achievements or hand over their problems, the answers from others will be stored in the same field, so the classification will base on ERP business fields, such as material requirements planning, capital requirements planning, bill of materials, purchase, inventory management, human resource, and so on[7]. This is the center part of business-oriented $\mathrm{KnS}$.

Considering the importance of knowledge to enterprise operation, we need to identify the restricted knowledge and give a sign in order to provide to certain group to use. Not all knowledge is public to all internal employees. Before storing them in 
databases, the knowledge should be identified by a qualified knowledge manager. It is necessary to do that if we want to make each of the databases useful.

Besides, we know that one of ERP advantages is to help enterprises change from mass production to mass customization production, so the customer's service will become the heart of enterprise operation. Managers and employees will pay much more attention to the knowledge related with customers' interest. This knowledge should be singled out before storing them in knowledge database according to certain attributes to the knowledge entity. In this way, a particular knowledge can be quickly selected out when needed.

\subsection{Knowledge Storage}

The knowledge provided in business-oriented $\mathrm{KnS}$ is almost a kind of formal knowledge, and it doesn't need strict checking. Most of the knowledge will be stored in database after classifying, indexing and describing. Because the existence of a variety of knowledge formats, there are different storage methods. In general, knowledge database should have two basic parts: data-based and document-based. Some knowledge forms, such as audio, video, and some word materials, should be stored into document-based part, but the description of these materials and the other knowledge should be stored into the relative database (data-based part). It is important to remember, however, that there must be a link between the document materials and their description records in relative database. That can insure the needed materials can be found quickly.

\subsection{Effective Evaluation Mechanism}

Many researchers have indicated that knowledge-sharing processes may fail if they are not backed by a supportive culture. But the culture can't be set up in one night. Without the culture, few employees would like to make their effort to exchange knowledge without any return. If an enterprise has no such culture environment, it is difficult to begin practicing business-oriented $\mathrm{KnS}$. Therefore, the effective evaluation mechanism becomes the key for successful implement of business-oriented $\mathrm{KnS}$. The key steps to effective application of business-oriented $\mathrm{KnS}$ at the beginning of tacit knowledge-sharing application are to develop supportive technology functions and build effective management mechanism. The following management mechanisms should be integrated into the tacit $\mathrm{KnS}$ system at the beginning of $\mathrm{KnS}$ practice.

- Feedback record function: Contentment to knowledge answer is the key criteria to evaluate the knowledge quality. Askers have their feeling to the answers of their question, and the feeling should be recorded by a certain method, such as giving scores, special signs, or evaluation.

- Effective identification mechanism: To identify person who provided much more individual knowledge than others will help top managers to better understand their workers, and the person may gain reputation as a knowledgeable source or receive the enterprise's rewards, such as promotion [8]. 
- General evaluation mechanism: Enterprises need to incorporate knowledgesharing proficiencies as part of annual job performance evaluations (as is already the case at Lenovo Company and Shanghai Mechanical Computer Company).

\section{SUBJECT-ORIENTED KNOWLEDGE-SHARING MODEL}

Subject-oriented knowledge-sharing flat is perceived as a replacement for a faceto-face interaction. During ERP implement, there are fewer opportunities for enterprise worker to make contact with others. Some researches have showed that the enterprise knowledge will only emerge as individual knowledge workers interact with other knowledge workers and the environment. Therefore, it is necessary for enterprises to make their efforts to build a platform for their workers to exchange their ideas. Though they still spend much more time facing their computers, they are keeping contact with their partner in cyberspace. The main function of the Subjectoriented knowledge-sharing flat is to create a friendly, freely and relax environment for their workers.

\subsection{Construction of Subject-oriented Knowledge-sharing Model}

In order to create a free and relax cyberspace for employees, the Subject-oriented knowledge-sharing needs to simulate the traditional natural communication environment. In natural environment, people have right to choose their partner(s), to choose their like topic and have right to keep their talk secret. Subject-oriented knowledge-sharing should have these functions, so the logic model of Subjectoriented knowledge sharing is shown as fig. 2 .

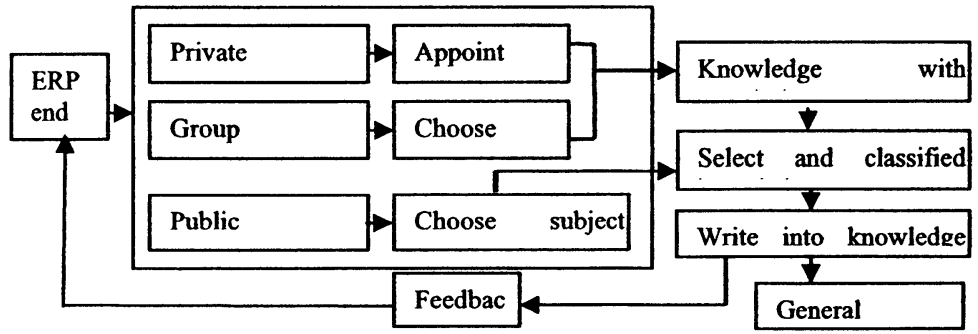

Figure 2. Subject-oriented KnS Process Model

From fig.2, we can see that there are three kinds of communication channels in Subject-oriented $\mathrm{KnS}$ process: private communication (which is between two persons), group communication (which is among a group, include teleconferencing), and public communication (which is to the public). The enterprise workers can freely choose one of them to interact with others or environment. Among the three channels, 
private and group communication should be kept secret, free and safe. Knowledge communication under this framework of $\mathrm{KnS}$ is just like the traditional face-to-face communication, and it refers to chat rooms. It allows workers to post their message publicly or privately. They post their public messages and questions on message board (to the public), or directly send to an appointed person (to individual), then wait for someone, either another worker or an expert, to answer the question. Sometimes it may take days to receive an answer, especially if one expects it to be answered by an expert. The public communication is a free field for anybody in an enterprise.

\subsection{Knowledge Classification and Storage}

Public communication is a place for workers to deliver their ideas to the public. In order to centralize the relative information, public communication fields should be divided into different subjects. The subjects should cover most of employs' interest, and they should be far beyond ERP business boundary, such as sport, travel, hot issues and so on. These subjects should be kept update according to the change. We can see the subjects are much different from that used in business-oriented $\mathrm{KnS}$.

Before storing into database, the knowledge should be selected seriously. For knowledge from public communication, the knowledge manager must first check whether it is of value, only value knowledge to enterprise operation can be further processed and stored into database. For knowledge from private or group communication field, the knowledge can be further processed only after received the permission or recommendation from participants in the group. Generally, two methods can be used for this work: computer programming and knowledge workers determining. The computer programming can judge the useful knowledge by evaluation, and special signs or scores that are provided by private or group communication participants. Compare to the computer programming, knowledge workers' decision has the flexibility and the veracity.

The process storage has two steps: first, communication content is recorded in temporary database and wait for checking; second, the knowledge that fits to enterprise demands is classified, indexed and then stored into formal database. After that, the knowledge can be provided to others. It is important to note that the private and the group process can be recorded only after getting their permission.

Unlike business-oriented $\mathrm{KnS}$, the practice process of subject-oriented $\mathrm{KnS}$ does not need much more external rewards. It is a natural process, and the motivation comes from human nature. In subject-oriented $\mathrm{KnS}$ model, there is no feedback or scores from the users. If the function is to be added, they are mainly to be used as an element to identify the value knowledge. This will be useful for computer programming to judge the value knowledge.

\section{CONCLUSIONS}

In this paper, two types of $\mathrm{KnS}$, business-oriented knowledge-sharing and subjectoriented knowledge-sharing, are discussed. There are both sides of $\mathrm{KnS}$ in ERP 
enterprise and support the two important functions: supporting business operation and employees' communication. Neither of these functions should be ignored. They are also important ways to get tacit knowledge from ERP end users and make tacit knowledge convert into explicit knowledge by IT technology support.

In recent years, more and more enterprises use ERP system in their main business process. Successful ERP system can greatly raise enterprise productivity. Meanwhile, many relevant researches and practices have showed that effective knowledge sharing can promote the competitive ability of enterprise. It is very important, therefore, to research the combination of $\mathrm{KnS}$ with ERP implementation. This paper has discussed the way to improve the integration of tacit $\mathrm{KnS}$ and ERP implementation in enterprises with the hope that it will help enterprise to effectively practice $\mathrm{KnS}$ in their ERP environment.

\section{REFERENCES}

1. M.C. Jones and R.L. Price, Organizational knowledge sharing in ERP implementation: Lessons from industry, Journal of organizational \& end user computing. Volume 16, Number 1, pp.21-40, (2004).

2. M.C. Jones, Tacit knowledge sharing during implementation: A multi-site case study, Information Resources Management Journal. Volume 18, Number 2, pp.1-23, (2005).

3. L. Wu, Y.W. Hsu, and C.S. Ong, ERP implementation: a quantitative model for organizational learning, International Journal of Information Technology \& Management. Volume 6, Number 1, p.5, (2007).

4. L.L. Hsu and M. Chen, Impacts of ERP systems on the integrated-interaction performance of manufacturing and marketing, Industrial management \& data systems. Volume 104, Number 1, pp.42-45, (2004).

5. A.T.Pardo, A.M. Cresswell, J. Zhang, and F. Thompson, Interorganizational knowledge sharing in public sector innovations, in Proc. of Academy of Management Proceedings, PNP: $A I$ (2001).

6. D.G. Bobrow and J. Whalen, Community knowledge sharing in practice: the Eureka story, Reflections. Volume 4, Number 2, pp.47-61, (2002).

7. Q. Chen, ERP-step Forward from Internal Integration, 2nd ed. (Publishing House of Electronics Industry: Beijing, 2006)

8. J. Liebowitz and Y. Chen, Developing knowledge-sharing proficiencies, Knowledge Management. Volume 3, Number 3, pp.12-17, (2004). 